\title{
NUMERICAL ANALYSIS OF ELASTIC PLATES WITH TWO OPPOSITE SIMPLY SUPPORTED ENDS BY SEGMENTATION METHOD
}

\author{
TARUN KanT $\dagger$ \\ Department of Civil Engineering, Indian Institute of Technology, Bombay 400076 , India
}

(Received 24 June 1980; received for publication 1 December 1980)

\begin{abstract}
A method for the numerical analysis of elastic plates with twn opposite simply supported ends is presented. A variety of boundary conditions including the mixed and the nonhomogeneous types can be prescribed along either of the remaining two opposite edges. Numerical results are presented for the three examples. Based on the comparisons made with the results available elsewhere, it is concluded that the present method is efficient, economical, reliable and very accurate.
\end{abstract}

\section{INTRODUCTION}

The present paper is concerned with the numerical analysis of rectangular plates with different boundary conditions. A less known formulation which is originally due to Goldberg et al.[1] is used in the present study. The system of equations are then numerically integrated by using a so-called "segmentation method" which is comprehensively documented in a recent publication [2]. This method is found to be efficient, reliable, accurate and computationally economical for a certain class of plate problems. The plate is assumed to be simply supported along two opposite edges. Different boundary conditions are then prescribed along the other two opposite edges.

\section{PRORLEM FORMULATION}

The present study is based on the thin plate theory [3] due to Kirchhoff with the following assumptions:

(1) Material is homogeneous, isotropic and linear elastic.

(2) Deflections are small compared to the thickness of the plate.

(3) Normals to the reference surface before deformation remain straight and normal to the deformed reference surface and their length remain unchanged $\left(\gamma_{x z}=\gamma_{y z}=\epsilon_{z}=0\right)$.

(4) Transverse normal stress components acting on planes parallel to the reference surface is neglected compared to other stress components $\left(\sigma_{z}=0\right)$.

The well-known governing equations of such a theory which defines a boundary value problem are summarised in Appendix A. Numerical integration of such a boundary value problem by the segmentation method [2] which is originally due to Goldberg et al.[1] involves first algebraic manipulation of the basic equations so as to obtain a set of first order differential equations-called the "intrinsic equations" involving only some particular dependent variables-called the "intrinsic variables", the number of which equals the order of the partial differential equation system of such a theory (fourth order in the present case). Then out of the two independent coordinates which describe the problem, one is chosen to be the preferred one. In the present analysis, $x$-coordinate is selected as the preferred one. Intrinsic equations are then derived consisting of a system of first

tAssistant Professor; Presently a Visitor at the Department of Civil Engineering, University of Wales, Swansea SA2 8PP (U.K.) under the Jawaharlal Nehru Memorial Trust (U.K.) Scholarship-1979 Award. order partial differential equations each of which contains necessarily the first derivative with respect of $x$ of one of the so-called intrinsic dependent variables which appear naturally in the boundary conditions on the edge $x=\mathrm{a}$ constant. In the present analysis, $Y$ defining the vector of intrinsic variables, consist of the dependent variables $w, \theta_{x}, V_{x}$ and $M_{x}$. After the required manipulations, the system of equations are obtained in the following form:

$$
\begin{aligned}
\frac{\partial w}{\partial x} & =-\theta_{x} \\
\frac{\partial \theta_{x}}{\partial x} & =\nu \frac{\partial^{2} w}{\partial y}+\frac{1}{D} M_{x} \\
\frac{\partial V_{x}}{\partial x} & =D\left(1-\nu^{2}\right) \frac{\partial^{2} w}{\partial y^{4}}-\nu \frac{\partial^{2} M_{x}}{\partial y^{2}}-\left(p_{z}^{+}+p_{z}^{-}+\rho h\right) \\
\frac{\partial M_{x}}{\partial x} & =-2 D(1-\nu) \frac{\partial^{2} \theta_{x}}{\partial y^{2}}+V_{x} .
\end{aligned}
$$

The other dependent variables are expressed as functions of intrinsic variables by simple algebraic relations, called "the auxiliary relations" in the following form:

$$
\begin{aligned}
& M_{y}=-D\left(1-\nu^{2}\right) \frac{\partial^{2} w}{\partial y^{2}}+\nu M_{x} \\
& M_{x y}=D(1-\nu) \frac{\partial \theta_{x}}{\partial y} \\
& Q_{x}=D(1-\nu) \frac{\partial^{2} \theta_{x}}{\partial y^{2}}+\frac{\partial M_{x}}{\partial x} \\
& Q_{y}=-D(1-\nu) \frac{\partial^{3} w}{\partial y^{3}}+\frac{\partial M_{x}}{\partial y} \\
& V_{y}=-D(1-\nu)^{2} \frac{\partial^{3} w}{\partial y^{3}}+(2-\nu) \frac{\partial M_{x}}{\partial y}
\end{aligned}
$$

The generalised displacement conponents and the corresponding stress resultants which form the vector $\underline{Y}$ of the intrinsic variables are functions of $x$ and $y$, and for a plate with two opposite edges, $y=0$ and $y=b$ as simply supported, these may be represented in the form of a Fourier series which automatically satisfies both the displacement and the force boundary conditions along these edges, to any desired degree of accuracy as follows:

$$
w(x, y)=\sum_{m} w_{m}(x) \sin (2 m-1) \frac{\pi y}{b}
$$




$$
\begin{aligned}
\theta_{x}(x, y) & =\sum_{m} \theta_{x m}(x) \sin (2 m-1) \frac{\pi y}{b} \\
V_{x}(x, y) & =\sum_{m} V_{x m}(x) \sin (2 m-1) \frac{\pi y}{b} \\
M_{x}(x, y) & =\sum_{m} M_{x m}(x) \sin (2 m-1) \frac{\pi y}{b} .
\end{aligned}
$$

Substitution of the expansions (3a-d) in the system of differential equations (la-d) and analytic integration of these equations with respect to the indpendent variable (coordinate) $y$ coupled with the use of the orthogonality conditions of the basic beam functions used in the $y$ direction in the aforesaid expansions reduces the set of partial differential equations (1a-d) into the following set of simultaneous first order ordinary differential equations (say for the $m$ th harmonic) involving only the four intrinsic variables. It may be mentioned that it is not necessary to express the external loads in the form of a Fourier series in the $y$-direction unlike the analytical[3] and the semi-analytical[4] methods. Further it is noted that the series uncouple with respect to the harmonic $m$ leading to a term-by-term analysis which enables storage of only the final discrete values of the intrinsic and the auxiliary dependent variables corresponding to a parti. cular harmonic analysis to be added to the values of the subsequent harmonic analyses.

$$
\begin{aligned}
\frac{\mathrm{d} w_{m} \dagger}{\mathrm{d} x}= & -\theta_{x m} \\
\frac{\mathrm{d} \theta_{x m}}{\mathrm{~d} x}= & -\nu(2 m-1)^{2}\left(\frac{\pi}{b}\right)^{2} w_{m}+\frac{1}{D} M_{x m} \\
\frac{\mathrm{d} V_{x m}}{\mathrm{~d} x}= & D\left(1-\nu^{2}\right)(2 m-1)^{4}\left(\frac{\pi}{b}\right)^{4} w_{m}+\nu(2 m-1)^{2} \\
& \times\left(\frac{\pi}{b}\right)^{2} M_{x m}-\frac{4}{\pi(2 m-1)^{2}}\left(p_{z}^{+}+p_{z}^{-}+\rho h\right) \\
\frac{\mathrm{d} M_{x m}}{\mathrm{~d} x}= & 2 D(1-\nu)(2 m-1)^{2}\left(\frac{\pi}{b}\right)^{2} \theta_{x m}+V_{x m}
\end{aligned}
$$

and the auxiliary relations $(2 \mathrm{a}-\mathrm{e})$ take the form:

$$
\begin{aligned}
M_{y}= & \sum_{m}\left[D\left(1-\nu^{2}\right)(2 m-1)^{2}\left(\frac{\pi}{b}\right)^{2} w_{m}\right. \\
& \left.+\nu M_{x m}\right] \sin (2 m-1) \frac{\pi y}{b} \\
M_{x y}= & \sum_{m} D(1-\nu)(2 m-1)\left(\frac{\pi}{b}\right) \theta_{x m} \cos (2 m-1) \frac{\pi y}{b} \\
Q_{x}= & \sum_{m}\left[D(1-\nu)(2 m-1)^{2}\left(\frac{\pi}{b}\right)^{2} \theta_{x m}\right. \\
& \left.+V_{x m}\right] \sin (2 m-1) \frac{\pi y}{b} \\
Q_{y}= & \sum_{m}\left[D(1-\nu)(2 m-1)^{2}\left(\frac{\pi}{b}\right)^{2} w_{m}+M_{x m}\right](2 m-1) \\
& \times\left(\frac{\pi}{b}\right) \cos (2 m-1) \frac{\pi y}{b} \\
V_{y}= & \sum_{m}\left[D(1-\nu)^{2}(2 m-1)^{2}\left(\frac{\pi}{b}\right)^{2} w_{m}+(2-\nu) M_{x n}\right] \\
& \times(2 m-1)\left(\frac{\pi}{b}\right) \cos (2 m-1) \frac{\pi y}{b}
\end{aligned}
$$

tFor the sake of brevity $w_{m}(x), M_{x m}(x)$, etc. are hereafter written simply as $w_{m}, M_{x m}$ etc.
The equations ( $4 a-d)$ are numerically integrated by the segmentation method [2] for th $m$ th harmonic at a time and the discrete point values of all the dependent variables are obtained by summing the corresponding values got for the given number of harmonics as given by the relations $(3 a-d)$ and $(5 a-e)$.

\section{NUMERICAL EXAMPLES}

Numerical results are presented for a square plate of side " $a$ " and thickness " $h$ ", simply supported along the two opposite edges, $y=0$ and $y=a$ with the boundary conditions $w_{m}=M_{y m}=0$ and loaded with a uniformly distributed load $p_{z}^{+}$. Discrete numerical values of the dependent variables are presented in the non-dimensional form as follows:

$$
\begin{aligned}
w & =\alpha \frac{p_{z}^{+} a^{4}}{D} \\
M_{x} & =\beta p_{z}^{+} a^{2} \\
M_{y} & =\beta_{1} p_{z}^{+} a^{2} \\
M_{x y} & =\frac{n}{2} p_{z}^{+} a^{2} \\
Q_{x} & =\gamma p_{z}^{+} a \\
Q_{y} & =\gamma_{1} p_{z}^{+} a \\
V_{x} & =\delta p_{z}^{+} a \\
V_{y} & =\delta_{1} p_{z}^{+} a .
\end{aligned}
$$

The same geometric and material properties, viz, $a / h=$ 50 and $\nu=0.3$ are used throughout. Five equal segments and five subdivisions within each segment for the Runge-Kutta-Gill algorithms [5] have been found suitable for the half plate analysis in the $x$-direction taking advantage of the symmetric conditions along the centre line in all the examples considered in this section. On an edge with outward unit normal vector $\hat{n}$ and unit tangent vector $t$, the following nomenclature has been used for the designation of the prescribed boundary conditons.

$$
\begin{gathered}
S \text { for } w=M_{n}=0 \\
C \text { for } w=\theta_{n}=0 \\
F \text { for } V_{n}=M_{n}=0 .
\end{gathered}
$$

Results obtained in the present study are compared with those available elsewhere [3]. Most of the discrete values of the dependent variables are tabulated for the first $\mathbf{2 0}$ harmonics. Convergence is seen to be excellent. It is observed that while the value of $w$ converges at the second or the third harmonic, the values of $M_{x}, M_{y}, M_{x y}$, $Q_{x}$ and $V_{x}$ take six to seven harmonics to converge. Convergence of the values of $Q_{y}$ and $V_{y}$ is seen to be slow.

Example 1. Plate with boundary conditions " $S$ " along all the four edges

The maximum values of $w, M_{x}, M_{y}, M_{x y}, Q_{x}, Q_{y}, V_{x}$ and $V_{y}$ are presented in Table 1 while the variation of $M_{x}$ and $M_{y}$ along the centre line $y / a=0.5$ is tabulated in Table 2. Variations of $Q_{x}$ and $V_{x}$ along the centre line $y / a=0.5$ and that of $M_{x y}, Q_{y}$ and $V_{y}$ along the supported edge $y / a=0.0$ are plotted in Figs. 1-3.

Example 2. Plate with boundary conditions " $C$ " along $x=0, a$ and " $S$ " along $y=0, a$ 
Table 1. Maximum values of transverse deflection and stress resultants in a square plate simply supported on all edges ( $w=M_{x}=0$ along $x=0, w=M_{y}=0$ along $y=0, a$ ) under U.D.L. based on Kirchhoff plate theory formulation of segmentation method-a convergence study $(\nu=0.3, a / h=50)$

\begin{tabular}{|c|c|c|c|c|c|c|c|c|}
\hline & $\begin{array}{l}w_{\max } \\
=\frac{p 2 a^{4}}{D}\end{array}$ & $\begin{array}{l}(M x)_{\max } \\
=B p z e^{2}\end{array}$ & $\begin{array}{l}\left(M_{\text {max }}\right. \\
=B_{1} p z a^{2}\end{array}$ & $\begin{array}{l}(M x y)_{\max } \\
=\frac{n}{2} p z a^{2}\end{array}$ & $\begin{array}{l}(Q x)_{\text {max }} \\
=\text { Yjza }\end{array}$ & $\begin{array}{l}(Q y)_{\text {max }} \\
=Y_{1} P z a\end{array}$ & $\begin{array}{l}(V x)_{\max } \\
=\delta p z a\end{array}$ & $\begin{array}{l}(V y)_{\text {max }} \\
=\delta_{1} p z a\end{array}$ \\
\hline$m$ & $\alpha$ & $B$ & $B_{1}$ & $\mathrm{n} / 2$ & $Y$ & $\gamma_{1}$ & $\delta$ & $\delta_{1}$ \\
\hline 1 & .00410 & .04915 & .05164 & -.03012 & .37145 & .24366 & .46612 & .32503 \\
\hline 2 & .00405 & .04760 & .04709 & -.03179 & .32646 & $.28 / 85$ & .40541 & למט/3. \\
\hline 3 & .00406 & .04791 & .04812 & -.03215 & .34266 & .30404 & .42728 & .38677 \\
\hline 4 & .00406 & .04780 & .04774 & -.03228 & .33440 & .31230 & .41612 & .39504 \\
\hline 5 & .00406 & .04785 & .04792 & -.03234 & .33939 & .31730 & .42287 & .40004 \\
\hline 6 & .00406 & .04783 & .04782 & -.03238 & .33605 & .32065 & .41835 & .40339 \\
\hline 7 & .00406 & .04784 & .04788 & -.03240 & .33844 & .32304 & .42159 & .40578 \\
\hline 8 & .00406 & .04783 & .04784 & -.03241 & .33604 & .32484 & .41910 & .40758 \\
\hline 9 & .00406 & .04784 & .04787 & -.03242 & .33805 & .32624 & .42105 & .40998 \\
\hline 10 & .00406 & .04783 & .04785 & -.03243 & .33692 & .32737 & .41954 & .41010 \\
\hline 15 & .00406 & .04784 & .04786 & -.03244 & .33765 & .33073 & .42052 & .41347 \\
\hline 20 & .00406 & .04784 & .04786 & -.03245 & .33730 & .33242 & .42004 & .41516 \\
\hline & $.00406^{a}$ & .0479 & .0479 & .0325 & .338 & .338 & .420 & .420 \\
\hline
\end{tabular}

avlues quoted in Ref. 3 based on Kirchhoff Plate Theory

Table 2. Bending moments in a square plate simply supported on all edges ( $w=M_{x}=0$ along $x=0, a ; w=M_{y}=0$ along $y=0, a$ ) under U.D.L. based on Kirchhoff plate theory formulation of segmentation method-a convergence study $(\nu=0.3, a / h=50)$

\begin{tabular}{|c|c|c|c|c|c|c|c|c|c|c|}
\hline \multirow[b]{2}{*}{$m$} & \multicolumn{5}{|c|}{$M x=B p z a^{2}, \frac{y}{a}=0.5$} & \multicolumn{5}{|c|}{$M y=\beta_{1} p z a^{2}, \frac{y}{a}=0.5$} \\
\hline & $\frac{x}{a}=0.1$ & $\frac{x}{a}=0.2$ & $\frac{x}{a}=0.3$ & $\frac{x}{a}=0.4$ & $\frac{x}{a}=0.5$ & $\frac{x}{a}=0.1$ & $\frac{x}{a}=0.2$ & $\frac{x}{a}=0.3$ & $\frac{x}{a}=0.4$ & $\frac{x}{a}=0.5$ \\
\hline 1 & .02211 & .03573 & .04372 & .04787 & .04915 & .01863 & .03314 & .04345 & .04960 & .05154 \\
\hline 2 & .02063 & .03403 & .04208 & .04630 & .04760 & .01633 & .02958 & .03926 & .04513 & .04709 \\
\hline 3 & .02099 & .03438 & .04240 & .04661 & .04791 & .01703 & .03052 & .04027 & .04615 & .04812 \\
\hline 4 & .02086 & .03476 & .04229 & .04650 & .04780 & .01673 & .03015 & .03989 & .04578 & .04774 \\
\hline 5 & .02092 & .03431 & .04234 & .04655 & .04785 & .01689 & .03033 & .04007 & .04595 & .04792 \\
\hline E & .02088 & .03428 & .04231 & .04652 & .04783 & -01680 & .03023 & .03997 & .04586 & .04782 \\
\hline 7 & .02090 & .03430 & .04233 & .04654 & .04784 & .01685 & .03029 & .04003 & .04592 & .04788 \\
\hline$\theta$ & .02089 & .03429 & .04232 & .04653 & .04783 & .01682 & .0302 & .03999 & .04588 & .04784 \\
\hline 9 & .02090 & .03430 & .04233 & .04654 & .04784 & .01684 & .03028 & .04002 & .04590 & .04787 \\
\hline 10 & .02089 & .03429 & .04232 & .04653 & .04783 & .01682 & .03026 & .04000 & .04588 & .04785 \\
\hline 15 & .02090 & .03429 & .04232 & .04653 & .04784 & .01683 & .03027 & .04001 & .04590 & .04786 \\
\hline 20 & .02090 & .03429 & .04232 & .04653 & .04784 & .01683 & .03026 & .04001 & .04589 & .04786 \\
\hline & $.0209^{3}$ & .0343 & .0424 & .0466 & .0479 & .0168 & .0303 & .0400 & .0459 & .0479 \\
\hline
\end{tabular}

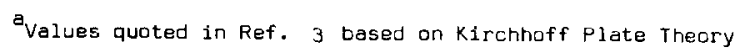




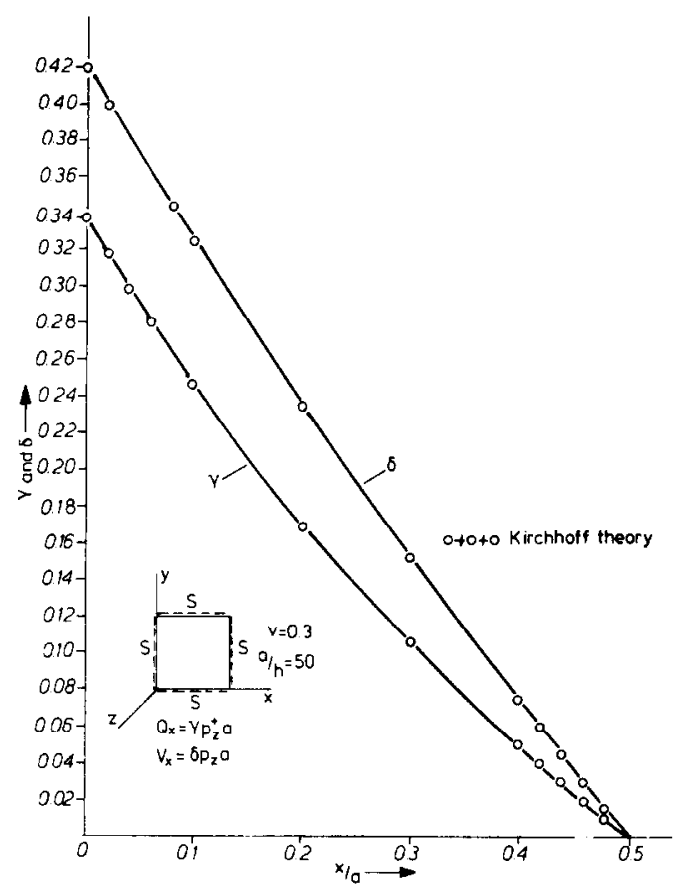

Fig. 1. Variation of $Q_{x}$ and $V_{x}$ along $y / a=0.5$.

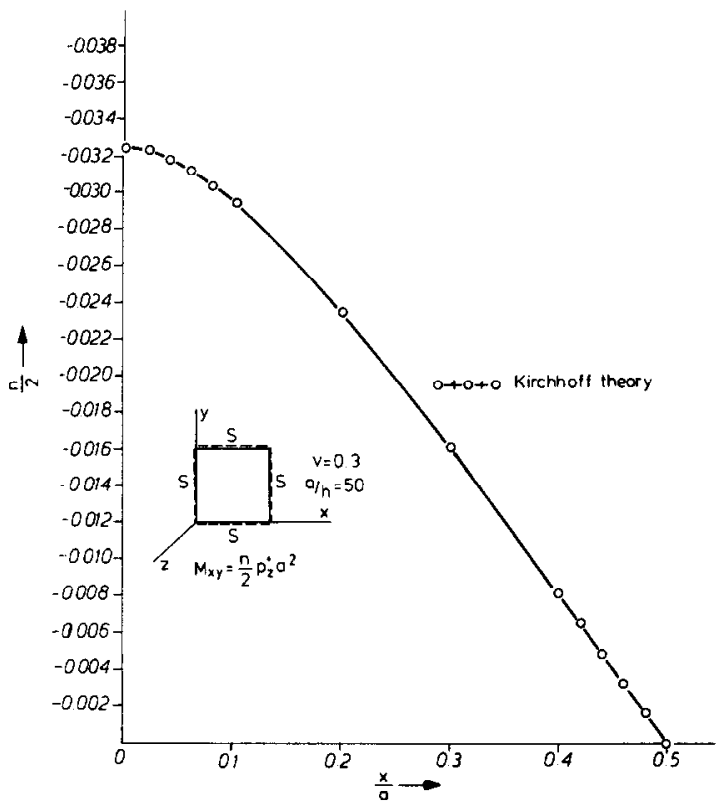

Fig. 2. Variation of $M_{x y}$ along the supported edge $(y / a=0)$.

Discrete numerical values of all the dependent variables are tabulated in Tables 3 and 4, while the variations of $Q_{x}, V_{x}, M_{x y}, Q_{y}$ and $V_{y}$ are plotted in Figs. 4-6. The occurrence of a maxima of $Q_{y}$ at the corner of the plate is unrealistic. But this is not due to the method used in the present study. This is due to the inherent limitation of the Kirchhoff plate theory itself. This point has been clarified in a recent paper[6].

Example 3. Plate with boundary conditions " $F$ " along $x=0, a$ and " $S$ " along $y=0, a$

Relevant discrete numerical values are presented in Tables 5 and 6. Plots of $Q_{x}$ and $V_{x}$ along the centre line

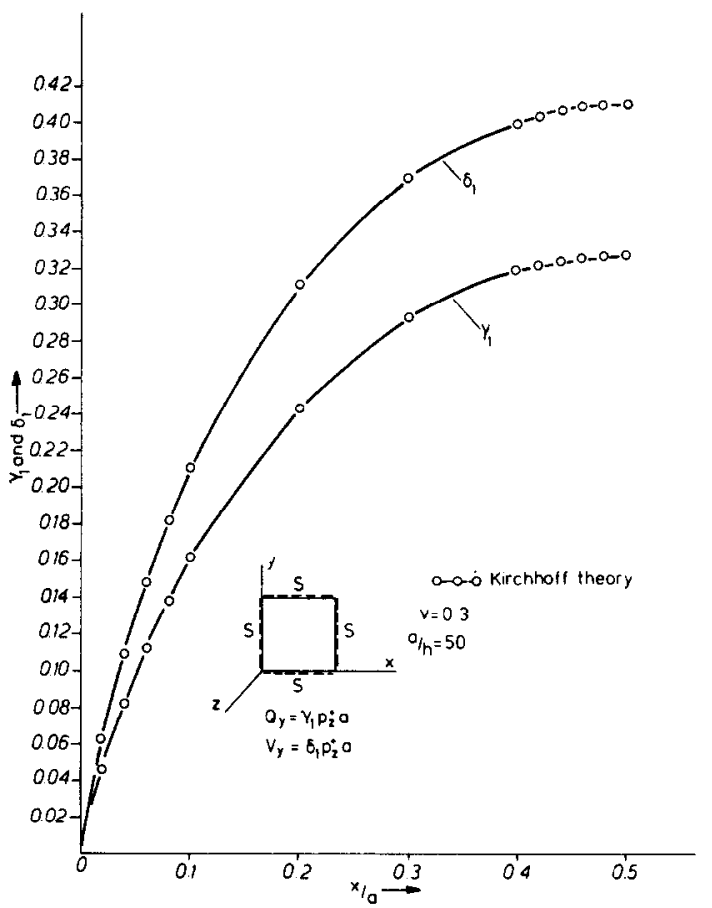

Fig. 3. Variation of $Q_{y}$ and $V_{y}$ along the supported edge $(y / a=$ 0).

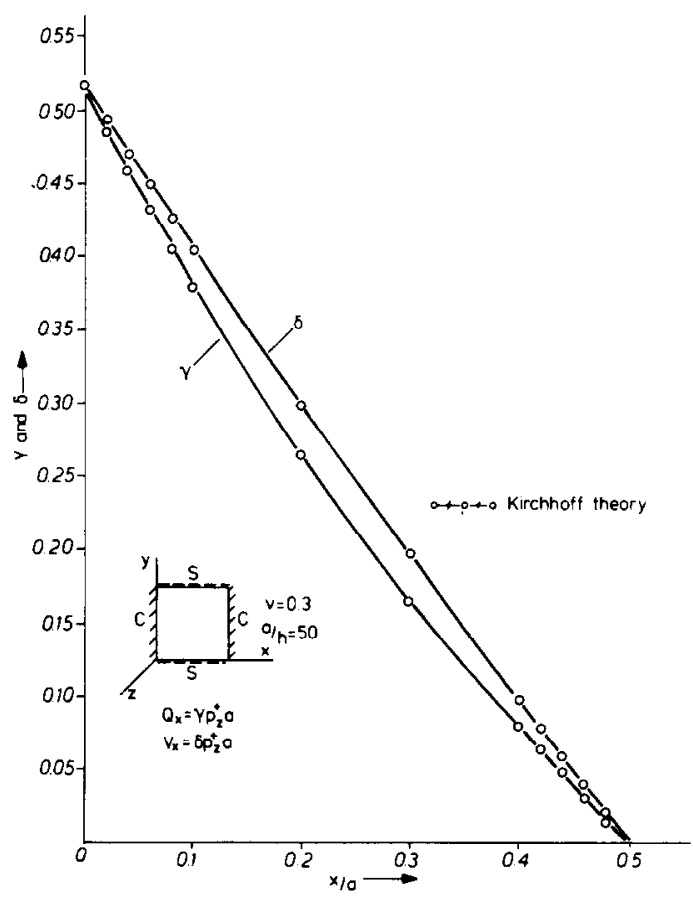

Fig. 4. Variation $Q_{x}$ and $V_{x}$ along $y / a=0.5$.

$y / a=0.5$, and $M_{x y}, Q_{y}$ and $V_{y}$ along the supported edge $y / a=0.0$ are shown in Figs. 7-9. These plots are quite revealing. It gives a feel for the variation of the quantities. The value of $Q_{x}$ is seen to be inconsistent at the free edge. This is due to the well-known problem of satisfaction of the boundary conditions at a free edge in a Kirchhoff plate, wherein a new quantity $V_{x}$ is introduced in the theory. In a recent publication[6] it is shown that barring its value at the free edge the variation of $Q_{x}$ is realistic in a physical situation. Thus it is 
Table 3. Maximum values of transverse deflection and stress resultants in a square plate with two opposite edges simply supported and the other two edges clamped ( $w=\theta_{x}=0$ along $x=0, a ; w=M_{y}=0$ along $\left.y=0, a\right)$ under U.D.L. based on Kirchhoff plate theory formulation of segmentation method-a convergence study $(\nu=0.3$, al $h=50)$

\begin{tabular}{|c|c|c|c|c|c|c|c|c|c|c|}
\hline m & $\begin{array}{l}w_{\max } \\
=\alpha \frac{p z a^{4}}{D}\end{array}$ & $\begin{array}{c}(M x)_{\max } \\
\text { Pos }\end{array}$ & $\begin{array}{c}=B P z \cdot a \\
\text { NEG }\end{array}$ & $\begin{array}{c}(M y)_{\max } \\
\text { POS }\end{array}$ & $\begin{array}{c}B_{\uparrow} p z a^{2} \\
\text { NEG }\end{array}$ & $\begin{array}{l}\left(M_{x y}\right)_{\text {max }}^{\dagger} \\
=\frac{n}{2} p z a^{2}\end{array}$ & $\begin{array}{l}(Q x)_{\max } \\
=Y D a\end{array}$ & $\begin{array}{l}(Q y)^{f+} \max \\
=v_{p} p z a\end{array}$ & $\begin{array}{l}(V x)_{\text {max }} \\
=\delta p z a\end{array}$ & $\begin{array}{l}(v y)^{f t} \max \\
=\delta_{1} p z^{\prime} a\end{array}$ \\
\hline & $\alpha$ & $B$ & $B$ & $B_{1}$ & $B_{1}$ & $n / 2$ & $\gamma$ & $Y_{1}$ & $\delta$ & $\delta_{1}$ \\
\hline 1 & .00196 & .03459 & -.07378 & .02800 & -.02213 & -.01332 & .58413 & -.23187 & .58413 & -.39418 \\
\hline 2 & .00191 & .03299 & -.06902 & .02352 & -.02071 & -.01426 & .49429 & -.27673 & .49429 & -.47044 \\
\hline 3 & .00192 & .03330 & -.07005 & .02464 & - .02102 & -.01436 & .52669 & -.29293 & .52669 & -.49798 \\
\hline 4 & .00192 & .03319 & -.06987 & .02427 & -.02090 & -.01437 & .51016 & -.30119 & .51016 & -.51203 \\
\hline 5 & .00192 & .03324 & -.06985 & .02444 & -.02096 & -.01438 & .52016 & -.30649 & .52016 & -.52053 \\
\hline$E$ & .00192 & .03321 & -.06975 & .02435 & -.02093 & -.01438 & .51347 & -.30954 & .51347 & -.52622 \\
\hline 7 & .00192 & .03323 & - .06981 & .02441 & -.02094 & -.01438 & .51826 & -.31194 & .51826 & -.53029 \\
\hline 8 & .00192 & .03322 & -.06978 & .02437 & -.02093 & -.01438 & .51466 & -.31374 & .51466 & -.53335 \\
\hline 9 & .00192 & .03322 & -.06980 & .02439 & -.02094 & -.01438 & .51746 & -.31514 & .51746 & -.53573 \\
\hline 10 & .00192 & .03322 & -.06978 & .02438 & -.02093 & -.01438 & .51522 & -.31626 & .51522 & -.53764 \\
\hline 15 & .00192 & .03322 & -.06979 & .02439 & -.02004 & -.01438 & .51667 & -.31963 & .51667 & -.54337 \\
\hline 20 & .00192 & .03322 & -.06979 & .02438 & -.02094 & -.01438 & .51597 & -.32131 & .51597 & -.54623 \\
\hline- & $.00192^{a}$ & & -.0697 & .0244 & & & & & & \\
\hline
\end{tabular}

Values quoted in Ref. 3 based on Kirchnoff Plate Theory

toccurs along the supported edge at $\frac{x}{\varepsilon}=0.2$ and $\frac{y}{a}=0$; ${ }^{t+}$ occurs at corners of the supported edges.

Table 4. Bending moments in a square plate with two opposite edges simply supported and the other two edges clamped ( $w=\theta_{x}=0$ along $x=0, a ; w=M_{y}=0$ along $\left.y=0, a\right)$ under U.D.L. based on Kirchhoff plate theory formulation of segmentation method-a convergence study $(\nu=0.3, a / h=50)$

\begin{tabular}{|c|c|c|c|c|c|c|c|c|c|c|c|c|}
\hline \multirow[b]{2}{*}{$m$} & \multicolumn{6}{|c|}{$M x=3 p z a^{2}, \quad y / a=0.5$} & \multicolumn{6}{|c|}{$M y=\beta_{1} p z a^{2}, \quad y / a=0.5$} \\
\hline & $\frac{x}{a}=0.0$ & $\frac{x}{a}=0.1$ & $\frac{x}{a}=0.2$ & $\frac{x}{a}=0.3$ & $\frac{x}{a}=0.4$ & $\frac{x}{a}=0.5$ & $\frac{x}{a}=0.0$ & $\frac{x}{a}=0.1$ & $\frac{x}{a}=0.2$ & $\frac{x}{a}=0.3$ & $\frac{x}{a}=0.4$ & $\frac{x}{a}=0.5$ \\
\hline 1 & -.07378 & -.02644 & .00364 & .02185 & .03155 & .03459 & -.02213 & -.00540 & .00875 & .01932 & .02581 & .02800 \\
\hline 2 & -.06902 & -.02669 & .00219 & .02020 & .02993 & .03299 & -.02071 & -.00652 & .00589 & .01551 & .02156 & .02362 \\
\hline 3 & -.07005 & -.02542 & .00253 & .02053 & .03024 & .03330 & -.02102 & -.00601 & .00676 & .01650 & .02258 & .02464 \\
\hline 4 & -.06967 & -.02654 & .00241 & .02042 & .03013 & .03319 & -.02090 & -.00628 & .00641 & .01612 & .02220 & .02427 \\
\hline 5 & -.06985 & -.02648 & .00247 & .02047 & .03018 & .03324 & -.02096 & -.00612 & .00658 & .01630 & .02238 & .02444 \\
\hline 6 & -.06975 & -.02652 & .00244 & .02044 & .03015 & .03321 & -.02093 & -.00621 & .00648 & .01620 & .02228 & .02435 \\
\hline 7 & -.06981 & -.02650 & .00246 & $.0204 E$ & .03017 & .03323 & -.02094 & -.00015 & .00654 & .01626 & .02234 & .02441 \\
\hline 8 & -.06978 & -.02651 & .00245 & .02045 & .03016 & .03322 & -.02093 & -.00619 & .00650 & .01622 & .02230 & .02437 \\
\hline 9 & -.06980 & -.02650 & .00245 & .02046 & .03017 & .03322 & -.02094 & -.00616 & .00653 & .01625 & .02233 & .02439 \\
\hline 10 & - .06978 & -.02651 & .00245 & .02045 & .03016 & .03322 & -.02093 & -.00618 & .00651 & .01623 & .02231 & .02438 \\
\hline 15 & -.06979 & -.02650 & .00245 & .02045 & .03017 & .03322 & -.02094 & -.00617 & .00652 & .01624 & .02232 & .02439 \\
\hline 20 & -.06979 & -.02650 & .00245 & .02045 & .03016 & .03322 & -.02094 & -.00618 & .00652 & .01624 & .02232 & .02438 \\
\hline & $-.0697^{a}$ & - & - & - & - & .0332 & - & - & - & - & - & .0244 \\
\hline
\end{tabular}

avalues quoted in Ref. 3 besed on Kirchhoff Plate Theory 
Table 5. Maximum values of transverse deflection and stress resultants in a square plate with two opposite edges simply supported and the other two edges free $\left(M_{x}=V_{x}=0\right.$ along $x=0, a ; w=M_{y}=0$ along $\left.y=0, a\right)$ under $(\nu=0.3$,

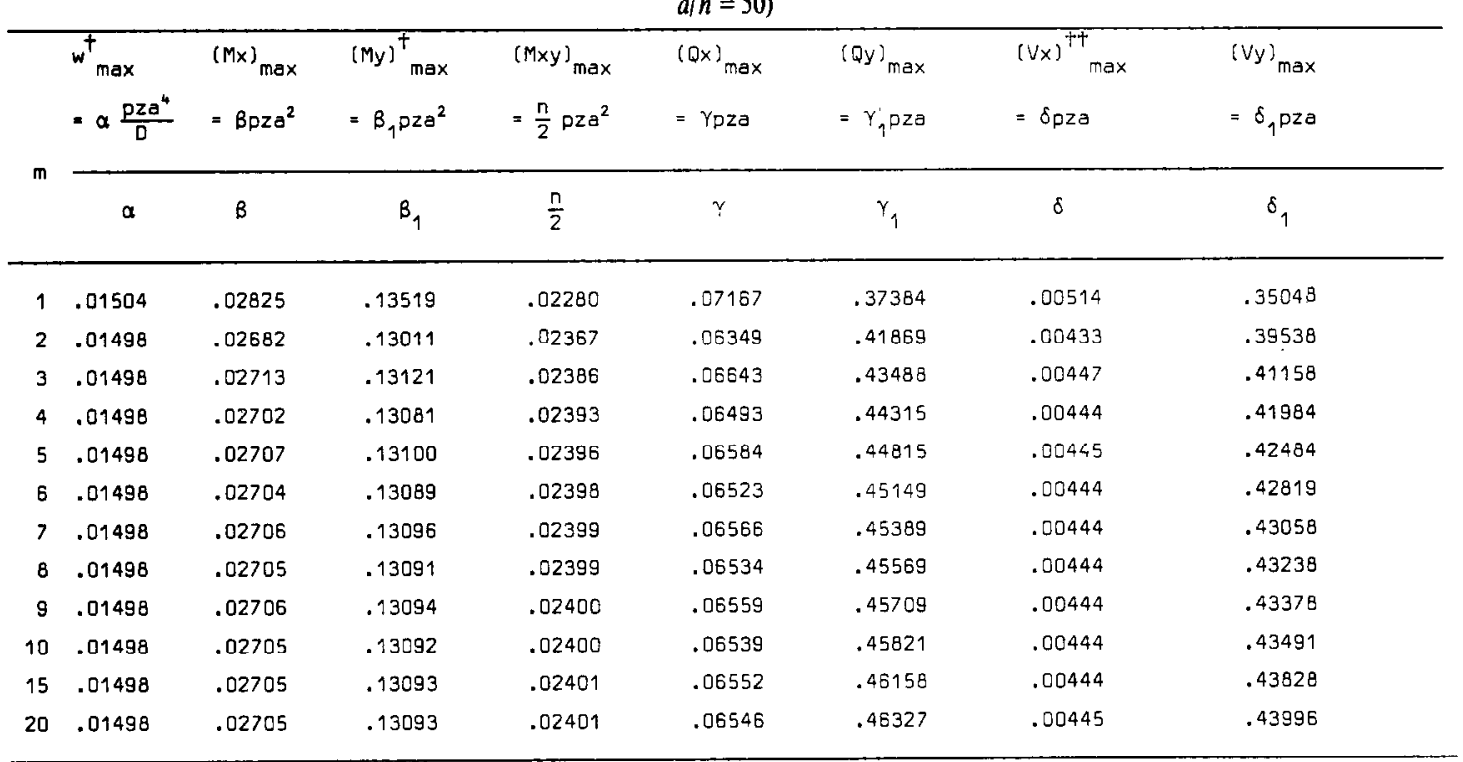

$$
\begin{aligned}
& t_{\text {Occurs at }} \frac{x}{a}=0 \text { and } \frac{y}{a}=0.5 \\
& +t_{\text {Occurs at }} \frac{x}{a}=0.2 \text { and } \frac{y}{a}=0.5
\end{aligned}
$$

Table 6. Bending moments in a square plate with two opposite edges simply supported and the other two edges free $V_{x}=M_{x}=0$ along $x=0, a ; w=M_{y}=0$ along $\left.y=0, a\right)$ under U.D.L. based on Kirchhoff plate theory formulation of

\begin{tabular}{|c|c|c|c|c|c|c|c|c|c|c|c|}
\hline \multirow{2}{*}{$\mathrm{m}$} & \multicolumn{5}{|c|}{$M x=B p z a^{2}, \quad y / a=0.5$} & \multicolumn{6}{|c|}{$M y=B_{1} p z a^{2}, \quad y / a=0.5$} \\
\hline & $\frac{x}{a}=0.1$ & $\frac{x}{a}=0.2$ & $\frac{x}{a}=0.3$ & $\frac{x}{a}=0.4$ & $\frac{x}{a}=0.5$ & $\frac{x}{a}=0.0$ & $\frac{x}{a}=0.1$ & $\frac{x}{a}=0.2$ & $\frac{x}{a}=0.3$ & $\frac{x}{a}=0.4$ & $\frac{x}{a}=0.5$ \\
\hline 1 & .01186 & .01972 & .02467 & .02739 & .02825 & .13519 & .13119 & .12875 & .12734 & .12660 & .12638 \\
\hline 2 & .01087 & .01842 & .02327 & .02596 & .02682 & .13011 & .12641 & .12402 & .12260 & .12186 & .12163 \\
\hline 3 & .01114 & .01872 & .02358 & .02627 & .02713 & .13121 & .12744 & .12505 & .12363 & .12280 & .12266 \\
\hline 4 & .01103 & .01861 & .02347 & .02616 & .02702 & .13081 & .12706 & .12467 & .12325 & .12251 & .12228 \\
\hline 5 & .01108 & .01866 & .02352 & .02521 & .02707 & .13100 & .12724 & .12485 & .12343 & .12269 & .12246 \\
\hline 6 & .01105 & .01863 & .02349 & .02618 & .02704 & .13089 & .12714 & .12475 & .12333 & .12259 & .12236 \\
\hline 7 & .01107 & .01865 & .02351 & .02620 & .02706 & .13096 & .12720 & .12481 & .12339 & .12265 & .12242 \\
\hline$B$ & .01106 & .01864 & .02350 & .02619 & .02705 & .13091 & .12716 & .12477 & .12335 & .12261 & .12238 \\
\hline 9 & .01107 & .01865 & .02351 & .02620 & .02706 & .13094 & .12719 & .12480 & .12338 & .12264 & .12241 \\
\hline 10 & .01106 & .01864 & .02350 & .02619 & .02705 & .13092 & .12717 & .12478 & .12336 & .12262 & .12239 \\
\hline 15 & .01106 & .01865 & .02350 & .02619 & .02705 & .13093 & .12718 & .12479 & .12337 & .12263 & .12240 \\
\hline 20 & .01106 & .01864 & .02350 & .02619 & .02705 & .13093 & .12718 & .12479 & .12337 & .12263 & .12240 \\
\hline
\end{tabular}
segmentation method-a convergence study $(\nu=0.3, a / h=50)$ 

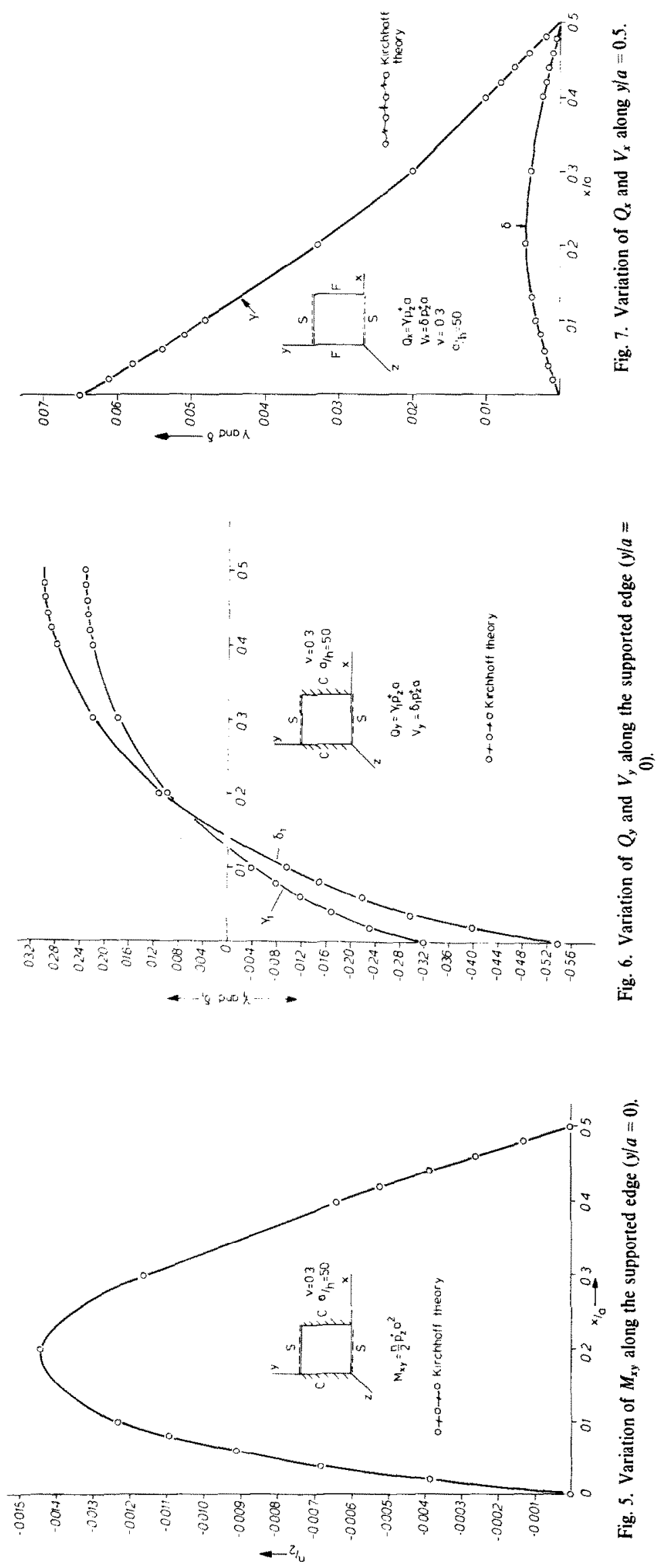


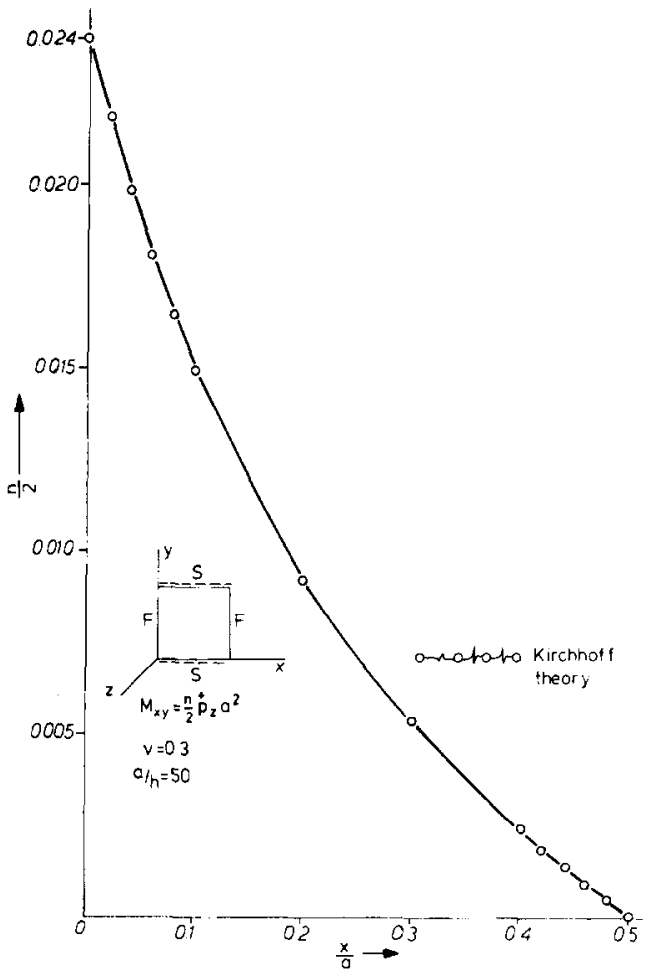

Fig. 8. Variation of $M_{x y}$ along the supported edge $(y / a=0)$.

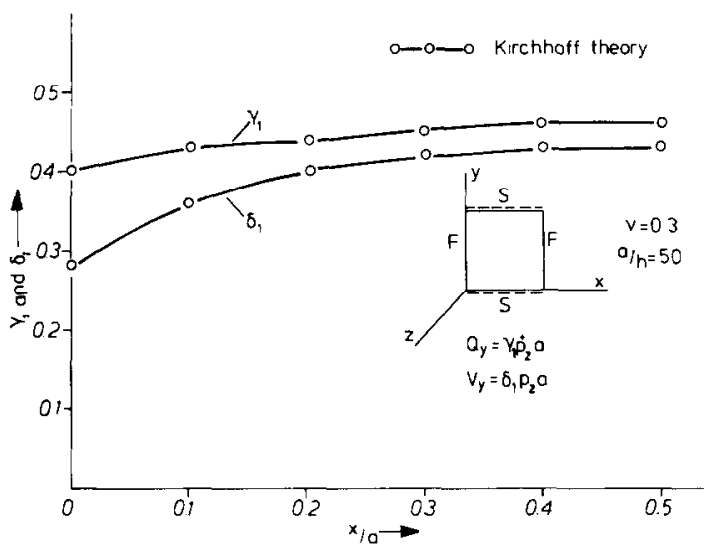

Fig. 9. Variation of $Q_{y}$ and $V_{y}$ along the supported edge ( $y / a=$ 0 ).

concluded that it is $Q_{x}$ and not $V_{x}$ which should be used in designs in such situations.

\section{CONCLUSIONS}

A less known formulation for economical numerical analysis of elastic plates is presented. The method is found to be very efficient and accurate. The numerical results obtained show excellent comparison with those of Timoshenko[3] which are presumably based on analytical solution. The main use of the method seems to lie in its adoption in design offices for preparation of reliable design charts at reasonable costs. Although the method has limitations in its applications to general problems when compared with the versatile finite element method [7], it appears to be superior to the finite strip method [4] because of its relatively accurate mathematical model.
Acknowledgements-The author is thankful to Dr. E. Hinton for helpful discussions. Sincere thanks are expressed to Mrs. Jean Davies for her very patient and excellent typing work. Initial support of this work by grants BRNS/ENGG/17/75 and HCS/DST/198/76 is gratefully acknowledged.

\section{REFERENCES}

1. J. E. Boldberg, A. V. Setlur and D. W. Alspaugh, Computer analysis of non-circular cylindrical shells. Proc., IASS Symp. Shell Structures in Engineering Practice, Budapest, Hungary (1965).

2. T. Kant and C. K. Ramesh, Numerical integration of linear boundary value problems in solid mechanics by segmentation method. Int. J. Numerical Methods Engng (to appear).

3. S. Timoshenko and S. Woinowsky-Krieger, Theory of Plates and Shells, 2nd Edn. McGraw-Hill, New York (1959).

4. Y. K. Cheung, Finite Strip Method in Structural Analysis. Pergamon Press, Oxford (1976).

5. A. Ralston and H. S. Wilf (Editors), Mathematical Methods for Digital Computers, pp. 95-120. Wiley, New York (1960).

6. T. Kant and E. Hinton, Analysis of rectangular Mindlin plates by the segmentation method, Report $C / R / 365 / 80$, Civil Engg. University of Wales, Swansea (1980).

7. O. C. Zienkiewicz, The Finite Element Method. McGraw-Hill, New York (1977).

\section{APPENDIX A}

Basic equations of Kirchhoff plate theory Displacement model (Ref. Fig. Al)

$$
\begin{gathered}
U(x, y, z)=z \theta_{x}(x, y) \\
V(x, y, z)=z \theta_{y}(x, y) \\
W(x, u, z)=w(x, y)
\end{gathered}
$$

Strain-displacement relations

$$
\begin{aligned}
& \epsilon_{x}=-z \frac{\partial^{2} w}{\partial x^{2}} \\
& \epsilon_{y}=-z \frac{\partial^{2} w}{\partial y^{2}} \\
& \gamma_{x y}=-2 z \frac{\partial^{2} w}{\partial x \partial y} \\
& \theta_{x}=-\frac{\partial w}{\partial x} \\
& \theta_{y}=-\frac{\partial w}{\partial y} .
\end{aligned}
$$

Equilibrium equations (Ref. Fig. A2-4)

$$
\frac{\partial Q_{x}}{\partial x}+\frac{\partial Q_{y}}{\partial y}+p_{z}^{+}+p_{z}^{-}+p h=0
$$

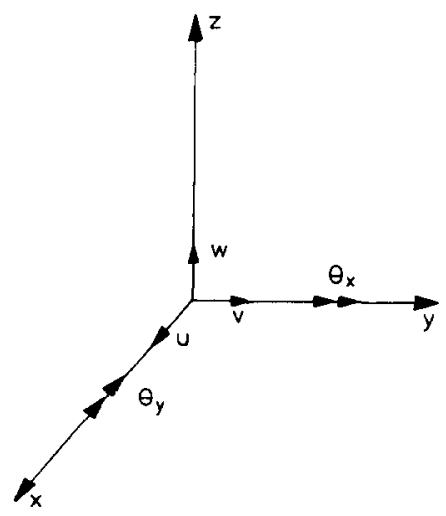

Fig. A1. Positive set of displacement components. 


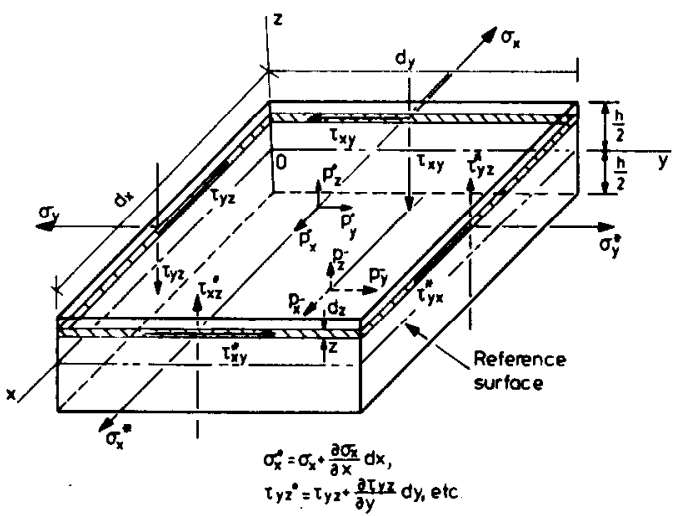

Fig. A2. Positive set of stress components.

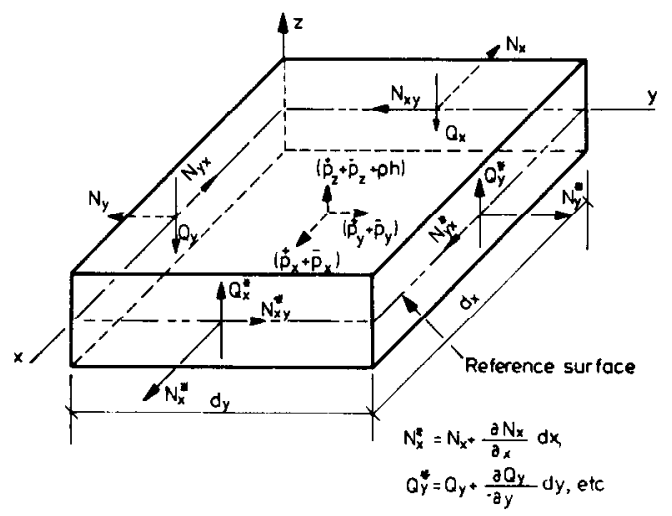

Fig. A3. Positive set of stress resultants-forces.

$$
\begin{aligned}
& \frac{\partial M_{x}}{\partial x}+\frac{\partial M_{x y}}{\partial y}-Q_{x}=0 \\
& \frac{\partial M_{x y}}{\partial x}+\frac{\partial M_{y}}{\partial y}-Q_{y}=0
\end{aligned}
$$

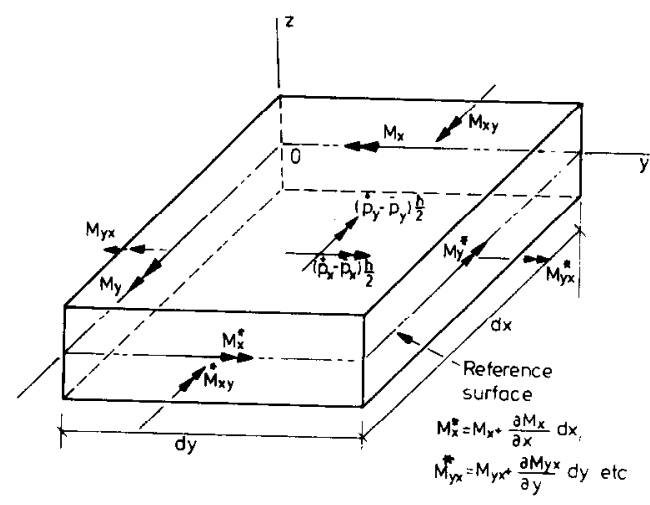

Fig. A4. Positive set of stress resultants-couples.

Force-displacement relations

$$
\begin{aligned}
& M_{x}=-D\left(\frac{\partial^{2} w}{\partial x^{2}}+\nu \frac{\partial^{2} w}{\partial y^{2}}\right) \\
& M_{y}=-D\left(\frac{\partial^{2} w}{\partial y^{2}}+\nu \frac{\partial^{2} w}{\partial x^{2}}\right) \\
& M_{x y}=-D(1-\nu) \frac{\partial^{2} w}{\partial x \partial y}
\end{aligned}
$$

\section{Kirchhoff shear and boundary conditions}

$V_{x}=Q_{x}+\frac{\partial M_{x y}}{\partial y}$

$V_{y}=Q_{y}+\frac{\partial M_{y x}}{\partial x}$

$w=\bar{w}$ or $\quad V_{x}=\bar{V}_{x}$

$\theta_{x}=\theta_{x}$ or $M_{x}=\bar{M}_{x}$

on edge $x=$ const. and

$$
\begin{array}{llll}
\omega=\bar{\omega} & \text { or } & V_{y}=\bar{V}_{y} \\
\theta_{y}=\bar{\theta}_{y} & \text { or } & M_{y}=\bar{M}_{y}
\end{array}
$$

on edge $y=$ const. 